\section{IN THIS ISSUE}

\section{Factor V Leiden testing to prevent pregnancy loss: different methods produce conflicting conclusions}

\section{see pages 335 and 374}

The search for an explanation for recurrent pregnancy loss (RPL) may include testing for genetic factors. Women who test positive for factor $\mathrm{V}$ Leiden mutation are at higher risk for RPL. Treatment may include aspirin and/or heparin, but it is unclear whether this treatment prevents future miscarriage. In this issue, Bajaj

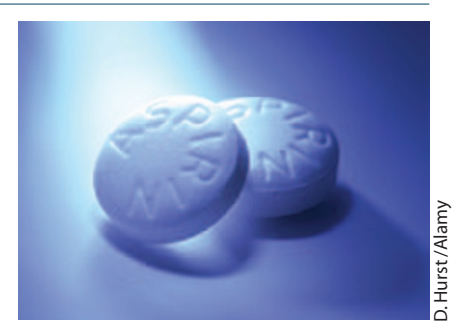
and Veenstra report that a decision model they developed suggests that factor V Leiden testing could result in small improvements in quality of life for women with RPL. Their position contrasts with the conclusions of a recent systematic review of two randomized clinical trials published in this journal in 2012. Those authors concluded that anticoagulation treatment does not improve pregnancy outcomes in women with RPL, except in the case of antiphospholipid antibody syndrome. In their related commentary, Grosse and Caughey offer an explanation for the seemingly contradictory conclusions, namely, that the two groups used different approaches to evidence analysis. Bajaj and Veenstra used a modeling approach based on observational data that allows them to address uncertainty. In addition, although their model suggests that factor V Leiden testing could be beneficial, Bajaj and Veenstra point out that the uncertainty of the underlying data limits their ability to assess the broader impact of testing and treatment on women and their families. - Karyn Hede, News Editor

\section{We screen newborns, don't we?}

\section{see page 332}

Newborn screening is a powerful example of how genetics can contribute to public health. Countless lives have been saved or dramatically improved, and vast expense to society averted, by identifying preventable diseases in newborns that would otherwise have resulted in serious morbidity or mortality.

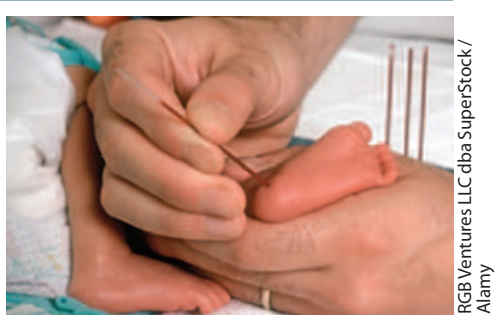

Now, because of technical advances in genomics, there exists another exciting possibility: targeted genomic screening of healthy adults to detect highly penetrant mutations for preventable conditions. For example, approximately 1 in 400 individuals in the United States carries a Lynch syndrome mutation, but we currently do not usually identify it until genetic testing is prompted by the development of cancer in the individuals or in their family members, or their death. If, instead, those at high risk were proactively identified via mutational analysis, considerable suffering could be averted. There exist effective preventive modalities for Lynch syndrome and several other genetic condi-

\section{NEWS BRIEFS}

\section{Large-scale sequencing solves mystery of dust mite origins}

House dust mites, those sneeze-inducing denizens of our mattresses and pillows, turn out to have a fascinating evolutionary history. Large-scale sequencing and

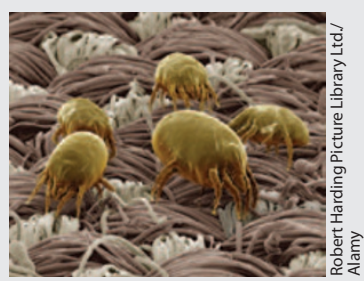
phylogenetic analysis recently revealed that they evolved from parasitic mites, which in turn evolved from free-living animals in the larger family of arachnids that also includes spiders. It is not fun to think about these eight-legged mites munching on one's sloughed skin, but the dust mite tells an interesting evolutionary tale, recently revealed via genome-scale comparative analysis. After testing 62 published hypotheses regarding the evolution of dust mites, researchers at the University of Michigan concluded that house dust mites evolved from the Psoroptidia, parasites that never leave the bodies of their hosts. Their immediate parasitic ancestors are livestock mange mites and ear mites living on dogs and cats. At some point, dust mites evolved to become free-living, a finding that runs counter to the long-held theory that obligate parasites don't return to free-living forms. Once people moved indoors, our dust mite companions found a feast of keratin in shed human skin and hair. Published 15 February 2013 in Systematic Biology, the study may assist research into the immune-response-triggering properties of dust mites that cause allergic symptoms in humans. The research may also lend insight into the ability of dust mites to inhibit host immune responses and thus eventually lead to new treatment for these allergies. - Karyn Hede, News Editor

\section{A GWAS shows that psychiatric disorders share genetic underpinnings}

Five major psychiatric disorders-autism, attention deficithyperactivity disorder, bipolar disorder, major depressive disorder, and schizophreniashare genetic risk factors, according to a large genome-wide association study (GWAS)

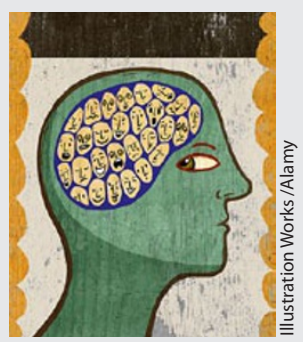
published 27 February 2013 in The Lancet. The identified molecular risk factors include four loci located in regions of chromosomes 3 p21 and 10q24, along with single-nucleotide polymorphisms (SNPs) in two genes encoding calcium channel blocker components. One of 


\section{IN THIS ISSUE}

tions, including predisposition to other specific cancers or to vascular catastrophe. Taking into consideration all such conditions, $0.5-1 \%$ of the US population - that is, millions of people in this country-harbor highly penetrant mutations for eminently preventable conditions.

In the commentary "We Screen Newborns, Don't We?," several of us, including colleagues from the world of public health, explore how we might sequence a carefully selected panel of genes in healthy individuals to identify at-risk individuals and prevent disease. We encourage a new partnership between geneticists and members of the public health community to investigate the feasibility of offering such screening to help realize the full promise of public health genomics. -James P. Evans, Editor-in-Chief

\section{NEWS BRIEFS}

the genes, CACNA1, had previously been linked to bipolar disorder and schizophrenia. The extensive study, conducted by the Psychiatric Genomics Consortium, compared the genomes of 33,332 patients and 27,888 controls. In addition, the study demonstrated that 14 SNPs previously significantly associated with schizophrenia and bipolar disorder have broader phenotypic effects. Overall, calcium channel activity was shown to play an important role in the development of all five disorders. Because these psychiatric disorders are treated clinically as distinct disorders, the findings assist the goal of moving beyond descriptive syndromes in psychiatry toward identifying and treating the root cause of disease. In an accompanying commentary, Alessandro Serretti and Chiara Fabbri, specialists in psychiatric disorders at the University of Bologna, Italy, concluded that the study will contribute to prediction and prevention of psychiatric diseases and provides new molecular targets for the next generation of psychotropic drugs. -Karyn Hede, News Editor

\section{Genetics in Medicine | Mission Statement}

Genetics in Medicine is a monthly journal committed to the timely publication of:

- Original reports which enhance the knowledge and practice of medical genetics

- Strategies and innovative approaches to the education of medical providers at all levels in the realm of genetics

As the official journal of the American College of Medical Genetics and Genomics (ACMG), the journal will:

- Provide a forum for discussion, debate and innovation concerning the changing and expanding role of medical genetics within the broader context of medicine

- Fulfill our responsibility to the College membership through the publication of guidelines, policy statements and other information that enhances the practice and understanding of medical genetics

Finally, as genetics becomes increasingly important in the wider medical arena, we will be an accessible and authoritative resource for the dissemination of medical genetic knowledge to providers outside of the genetics community through appropriate reviews, discussions, recommendations and guidelines. 